\title{
Enhanced recovery after thoracic surgery is associated with improved adjuvant chemotherapy completion for non-small cell lung cancer
}

\author{
David B. Nelson, MD, MSc, Reza J. Mehran, MD, Kyle G. Mitchell, MD, Arlene M. Correa, PhD,
} Boris Sepesi, MD, Mara B. Antonoff, MD, and David C. Rice, MB, BCh

\section{ABSTRACT}

Objective: Enhanced recovery after surgery integrates multiple evidence-based interventions to accelerate postoperative recovery. We hypothesized that enhanced recovery after surgery would also facilitate adjuvant chemotherapy for non-small cell lung cancer.

Methods: An enhanced recovery after surgery pathway was implemented at a single institution, starting with a transitional rollout in 2012 and full rollout in 2015. Patients with clinical stage I or II non-small cell lung cancer without induction therapy and who were pathologic nodal positive or had $5-\mathrm{cm}$ or larger tumor size were selected for analysis. Dates analyzed were pre-enhanced recovery after surgery (2006-2011), transitional (2012-8/2015), and enhanced recovery after surgery (9/2015-2017). Interrupted time series was used to analyze trends in time to receive adjuvant chemotherapy after resection by era. Logistic regression was used to determine factors associated with receiving 4 or more cycles of adjuvant chemotherapy.

Results: A total of 471 patients were identified. The interval between lung resection and commencing adjuvant chemotherapy was progressively shorter after the transition era $(P=.041)$. The rate of receiving adjuvant chemotherapy progressively increased (from $40 \%$ pre-enhanced recovery after surgery, to $50 \%$ transition era, to $62 \%$ enhanced recovery after surgery era, $P<.001)$. Multivariable regression revealed the enhanced recovery after surgery era (odds ratio, 3.6, $P<.001$ ), the transitional era (odds ratio, $2.01, P=.007$ ), $\mathrm{pN}$ status, tumor grade and histology, age, and preoperative performance status were associated with completing adjuvant therapy. The surgical approach, whether open or thoracoscopic, was not associated with completing adjuvant chemotherapy.

Conclusions: Enhanced recovery after surgery was associated with facilitated delivery of adjuvant chemotherapy, with a shortened interval to receive adjuvant chemotherapy and a higher rate of receiving 4 or more cycles. (J Thorac Cardiovasc Surg 2019;158:279-86)

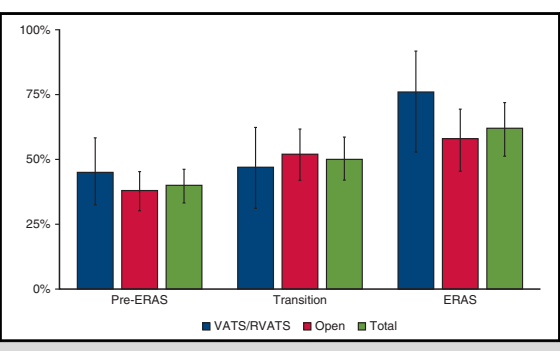

Adjuvant chemotherapy use by era

\section{Central Message}

After implementation of an enhanced recovery program, patients started adjuvant chemotherapy earlier and were more likely to complete at least 4 cycles of therapy.

\section{Perspective}

Enhanced recovery programs are associated with improvement in many short-term outcomes, including postoperative morbidity, pain, length of stay, and overall patient satisfaction. We demonstrate that enhanced recovery was also associated with improvement in adjuvant chemotherapy use.

See Commentary on page 287.

\footnotetext{
From the Department of Thoracic and Cardiovascular Surgery, The University of Texas MD Anderson Cancer Center, Houston, Tex.

This study was funded by generous philanthropic donations from the Mason Family, anonymous donors, and departmental support.

Read at The American Association for Thoracic Surgery Thoracic Summit, New York, New York, October 12-13, 2018.

Institutional Review Board Protocol PA15-0180, Revision Approval Date March 8, 2018.

Received for publication Oct 9, 2018; revisions received Feb 5, 2019; accepted for publication March 2, 2019; available ahead of print April 20, 2019.

Address for reprints: David C. Rice, MB, BCh, Thoracic and Cardiovascular Surgery, The University of Texas MD Anderson Cancer Center, 1400 Pressler St, Unit 1489, Houston, TX 77030 (E-mail: drice@mdanderson.org). 0022-5223/\$36.00

Copyright (c) 2019 Published by Elsevier Inc. on behalf of The American Association for Thoracic Surgery

https://doi.org/10.1016/j.jtcvs.2019.03.009
}

Lung resection can be a morbid operation, with up to $40 \%$ of patients experiencing a postoperative complication. Enhanced recovery after surgery (ERAS) pathways consist of multiple evidence-based protocols that aim to diminish surgical stress and accelerate postoperative recovery. ${ }^{2}$

Scanning this QR code will
take you to the article title
page to access supplementary
information.




$$
\begin{aligned}
& \text { Abbreviations and Acronyms } \\
& \begin{aligned}
\text { CI } & =\text { confidence interval } \\
\text { ECOG } & =\text { Eastern Cooperative Oncology Group } \\
\text { ERAS } & =\text { enhanced recovery after surgery } \\
\text { FEV1 } & =\text { predicted forced expiratory volume in } \\
& 1 \text { second } \\
\text { NSCLC }= & \text { non-small cell lung cancer } \\
\text { OR } & =\text { odds ratio } \\
\text { VATS } & =\text { video-assisted thoracoscopic surgery }
\end{aligned}
\end{aligned}
$$

These pathways include early ambulation, reduction of opioid use with multimodal analgesia, protocolized fluid and drain management, and nutritional optimization. ${ }^{3}$ After implementing ERAS, multiple centers have reported decreased postoperative complications, improved pain relief, shortened hospital stays, and increased patient satisfaction after lung resection. ${ }^{4-13}$ We hypothesized that ERAS would also facilitate return to intended oncologic treatment, such as adjuvant chemotherapy, which is considered the standard of care for surgically resected stage II and greater non-small cell lung cancer (NSCLC).

Most reports on ERAS are nonrandomized because of the challenges with implementing complex changes in surgical care. ${ }^{4}$ To establish safety and feasibility, 2 thoracic surgeons trialed components of ERAS during a transitional era from January 1, 2012, to August 31, 2015. ERAS was fully implemented by September 1, 2015. We used the quasiexperimental interrupted time series approach to analyze differences in the time to receive adjuvant chemotherapy pre- and post-ERAS implementation. ${ }^{14-17}$ Because the clinical importance of earlier delivery of adjuvant chemotherapy has not been well demonstrated, ${ }^{18,19}$ we also explored whether ERAS implementation was associated with improvements in completing 4 or more cycles of adjuvant chemotherapy.

\section{MATERIALS AND METHODS Patient Selection}

This study was approved by the Institutional Review Board at The University of Texas MD Anderson Cancer Center, which waived the requirement for informed consent. A prospectively maintained departmental database was queried to identify patients who had undergone lung resection for NSCLC from January 1, 2006, to December 31, 2017. Patients with clinical stage I or II NSCLC who had not received induction chemotherapy or radiotherapy and were found to have pathologic nodal involvement or 5$\mathrm{cm}$ or larger tumor size after lung resection were selected for study inclusion. These patients would qualify for receipt of adjuvant chemotherapy per National Comprehensive Cancer Network guidelines. ${ }^{20}$ All patients underwent tumor resection with curative intent.

\section{Implementation of Enhanced Recovery After Surgery Protocol}

Characteristics of protocolized patient care during each era are detailed within the Appendix E1 and summarized in Table 1. ${ }^{21}$

\section{Data Collection and Variable Definitions}

All covariates were collected and maintained in a prospectively maintained departmental database. Outpatient postoperative performance status was prospectively recorded by clinic staff and reflected performance status on the day of the patient visit. Charts were reviewed to ascertain the number of cycles of adjuvant chemotherapy received at our institution or at an outside institution. Dates of chemotherapy delivery were collected through a structured institutional data search and were available for 175 patients who received chemotherapy. Cardiac and pulmonary complications were defined as previously described in a recent publication by our group. ${ }^{11}$

\begin{tabular}{|c|c|c|}
\hline & Pre-ERAS & ERAS \\
\hline \multicolumn{3}{|l|}{ Preoperative } \\
\hline Education & Video & Additional teaching \\
\hline Preoperative fasting & $8 \mathrm{~h}$ & $2 \mathrm{~h}$ \\
\hline Preanesthetic analgesia & Absent & Tramadol/gabapentin \\
\hline \multicolumn{3}{|l|}{ Intraoperative } \\
\hline Perioperative steroids & Absent & Dexamethasone \\
\hline Analgesia & Opioids & Acetaminophen, ketorolac \\
\hline Anesthesia & Volatile & Total intravenous \\
\hline Fluid replacement & Fixed & Goal directed algorithm based on stroke volume variation \\
\hline Regional analgesia & Epidural & Long-acting liposomal bupivacaine intercostal nerve block \\
\hline \multicolumn{3}{|l|}{ Postoperative } \\
\hline Analgesia & Patient controlled analgesia (opioid pump/epidural) & Acetaminophen, ketorolac, gabapentin with opioids as needed \\
\hline Early ambulation & Routine & Routine \\
\hline Early oral intake & Clear liquids on POD 1 & Clear liquids on POD 0 \\
\hline Chest drains & Two $28 \mathrm{~F}-32 \mathrm{~F}$ & Single $20 \mathrm{~F}-24 \mathrm{~F}$ \\
\hline
\end{tabular}
Receipt of planned adjuvant chemotherapy was defined as receiving 4 or more cycles of adjuvant chemotherapy within 180 days of surgery. The seventh edition of the AJCC Cancer Staging Manual was used to define the clinical stage. Patients who were converted to a thoracotomy after videoassisted thoracoscopic surgery (VATS) or robotic-assisted thoracoscopic surgery were counted as a thoracotomy for the purposes of this analysis. In cases of missing data for tumor grade $(n=15 / 471)$, the tumor grade

TABLE 1. Enhanced recovery after surgery protocol

ERAS, Enhanced recovery after surgery; $P O D$, postoperative day. 
was set to median (moderate differentiation). In cases of missing data for percent of predicted forced expiratory volume in 1 second (FEV1) $(n=28)$, the missing data were set to median $(83 \%$ of predicted).

\section{Statistical Methods}

The chi-square or Fisher exact test was used to compare frequency of categoric variables from pre-ERAS to transition to ERAS era. The Mantel-Haenszel test was used to analyze heterogeneity in adjuvant chemotherapy use by thoracoscopic or open approaches. The KruskalWallis test was used to evaluate differences in continuous variables in each time period.

To determine differences in postoperative performance status by each era, a mixed-effects ordered logistic regression model was created with a patient-specific random intercept. The regression model was selected a priori and included age, surgical approach, extent of resection, FEV1, preoperative performance status, gender, and the postoperative month as covariates. Analysis was restricted to postoperative performance status recorded within the first year. The Kaplan-Meier method with the log-rank test was also used to determine differences in time to event analysis, with the event being defined as Eastern Cooperative Oncology Group (ECOG) performance status 0 during follow-up clinic visits.

An interrupted time series analysis was used to determine changes in the time to onset of adjuvant chemotherapy before and after ERAS was implemented. A time series averages a sequence of values taken during regular intervals and compares the level and trend before and after intervention. In this case, the median time to onset of chemotherapy was measured per month during the entire study duration. Autocorrelation was explored with use of the Cumby-Huizinga test with up to 12 lags tested and was not found to be present. ${ }^{22}$

A univariable logistic regression model was used to evaluate factors associated with receipt of adjuvant chemotherapy, which was defined as 4 cycles or more. Covariates with $P$ value less than .25 or those of clinical interest were entered into a multivariable analysis. Covariates analyzed included age, extent of resection, ERAS era, surgical approach, pathologic $\mathrm{T}$ and $\mathrm{N}$ status, American Society of Anesthesiologists status, preoperative performance status, percent predicted FEV1, tumor grade, and tumor histology. A sensitivity analysis excluding sublobar resections was performed without a change in results. All tests were 2-sided. All statistical analyses were performed with STATA version 14.2 (StataCorp LP, College Station, Tex).

\section{RESULTS}

\section{Patient Characteristics}

A total of 471 patients met inclusion criteria. From the pre-ERAS era to the ERAS era, there were no trends identified among preoperative characteristics, including tumor histology or grade, pathologic stage, preoperative performance status, extent of resection, or surgical approach (Table 2). Cardiopulmonary complications decreased from $41 \%$ during pre-ERAS to $34 \%$ during the transition era and to $25 \%$ after ERAS implementation $(P=.025)$, findings that have been published by our group. ${ }^{11}$

\section{Improved Postoperative Performance Status With Enhanced Recovery}

More patients were documented in the clinic as being ECOG performance status 0 earlier during the ERAS era compared with the pre-ERAS era (Figure $1, P=.001$ ). The adjusted proportional odds ratio (OR) of a unit increase in postoperative performance status during the transition era was $0.71(95 \%$ confidence interval [CI], 0.45-1.17; $P=.185)$ compared with the pre-ERAS era. However, during the ERAS era, the adjusted proportional OR of a unit increase in postoperative performance status compared with the pre-ERAS era was $0.38 \quad(95 \%$ CI, $0.18-0.81$; $P=.013$ ), indicating the enhanced recovery era was associated with improved postoperative performance status recovery.

\section{Earlier Use of Adjuvant Chemotherapy After Enhanced Recovery After Surgery Implementation}

During the pre-ERAS era (2006-2011), the interval from surgery to receipt of adjuvant chemotherapy was stable over time $(P=.953)$ (Figure 2$)$. When ERAS was partially implemented in 2012, there was also no immediate change in the time to receive adjuvant chemotherapy $(P=.364)$. However, there was a statistically significant interaction between the slope before and after commencement of the ERAS transition period $(P=.041)$, indicating that patients were receiving adjuvant chemotherapy earlier after implementing enhanced recovery.

\section{Rate of Completing Adjuvant Chemotherapy by Era}

During the pre-ERAS era, the rate of completing 4 or more cycles of adjuvant chemotherapy was $40 \%$ (Table 3). The rate increased to $50 \%$ during the transition era and to $62 \%$ during the ERAS era $(P<.001)$. Both minimally invasive and open approaches showed improvements in adjuvant chemotherapy use from the pre-ERAS to ERAS era, with no observed evidence of heterogeneity $(P=.308)$.

\section{Factors Associated With Receiving Full Adjuvant Chemotherapy}

Older patients were less likely to receive adjuvant chemotherapy, with those who were aged 65 to 74 years (OR, 0.37; 95\% CI, $0.22-0.61 ; P<.001$ ) or more than 75 years (OR, $0.10 ; 95 \% \mathrm{CI}, 0.05-0.18 ; P<.001)$ showing markedly decreased use on multivariable regression (Figure 3). Patients with a preoperative performance status of 1 or greater were also less likely to receive adjuvant chemotherapy (OR, 0.53; 95\% CI, 0.33-0.84; $P=.007$ ). Bilobectomy or pneumonectomy was associated with decreased adjuvant chemotherapy use compared with a lobectomy (OR, $0.41 ; 95 \% \mathrm{CI}, 0.21-0.79 ; P=.008)$. The rate of receiving adjuvant chemotherapy also varied by indication, with tumor grade, histology, and pathologic status being associated with adjuvant chemotherapy. Adjuvant chemotherapy improved during the transition era (OR, 2.01; 95\% CI, 1.21-3.34; $P=.007)$ and the ERAS era (OR, 3.55; 95\% CI, 1.94-6.52; $P<.001$ ) compared with the pre-ERAS era. However, surgical approach, whether by open or minimally invasive approaches, was not associated with receiving complete adjuvant chemotherapy. 
TABLE 2. Patient, tumor, and operative characteristics

\begin{tabular}{|c|c|c|c|c|}
\hline & $\begin{array}{c}\text { Pre-ERAS } * \\
(\mathbf{N}=\mathbf{2 3 0})\end{array}$ & $\begin{array}{c}\text { Transition* } \\
(\mathrm{N}=149)\end{array}$ & $\begin{array}{c}\text { ERAS* } \\
(\mathbf{N}=\mathbf{9 2})\end{array}$ & $P$ value \\
\hline Age (y) & $66(59-73)$ & $66(58-75)$ & $66(58-73)$ & .968 \\
\hline \multicolumn{5}{|l|}{ Sex } \\
\hline Male & $126(55 \%)$ & $76(51 \%)$ & $43(47 \%)$ & .408 \\
\hline FEV1 ( $\%$ of predicted $) \dagger$ & $83(70-97)$ & $84(72-98)$ & $82(72-98)$ & .816 \\
\hline Preoperative performance status & & & & .567 \\
\hline 0 & $145(63 \%)$ & $103(69 \%)$ & $60(65 \%)$ & \\
\hline 1 & $84(37 \%)$ & $46(31 \%)$ & $31(34 \%)$ & \\
\hline 2 & $1(<1 \%)$ & 0 & $1(1 \%)$ & \\
\hline ASA score & & & & .145 \\
\hline 2 & $23(10 \%)$ & $10(7 \%)$ & $4(4 \%)$ & \\
\hline 3 & $206(90 \%)$ & $135(91 \%)$ & $87(95 \%)$ & \\
\hline 4 & $1(0.4 \%)$ & $4(3 \%)$ & $1(1 \%)$ & \\
\hline Histology & & & & .532 \\
\hline Adenocarcinoma & $139(60 \%)$ & $93(62 \%)$ & $56(61 \%)$ & \\
\hline Squamous cell & $67(29 \%)$ & $37(25 \%)$ & $21(23 \%)$ & \\
\hline Other & $24(10 \%)$ & $19(13 \%)$ & $15(16 \%)$ & \\
\hline Tumor grade $\ddagger$ & & & & .151 \\
\hline Well & $29(13 \%)$ & $19(13 \%)$ & $10(11 \%)$ & \\
\hline Moderate & $112(49 \%)$ & $72(49 \%)$ & $58(64 \%)$ & \\
\hline Poor & $83(36 \%)$ & $52(35 \%)$ & $19(21 \%)$ & \\
\hline Undifferentiated & $1(<1 \%)$ & 0 & $1(1 \%)$ & \\
\hline Pathologic stage & & & & .300 \\
\hline IIA & $86(37 \%)$ & $57(38 \%)$ & $39(42 \%)$ & \\
\hline IIB & $37(16 \%)$ & $36(24 \%)$ & $19(21 \%)$ & \\
\hline IIIA & $99(43 \%)$ & $55(37 \%)$ & $34(37 \%)$ & \\
\hline IIIB & $4(2 \%)$ & 0 & 0 & \\
\hline IV & $4(2 \%)$ & $1(1 \%)$ & 0 & \\
\hline Tumor size, $\mathrm{cm}$ & $3.5(2-5.3)$ & $3.9(2.8-5.3)$ & $3.5(2.4-6)$ & .125 \\
\hline Resection & & & & .628 \\
\hline Sublobar & $17(7 \%)$ & $7(5 \%)$ & $3(3 \%)$ & \\
\hline Lobectomy & $178(78 \%)$ & $120(81 \%)$ & $74(80 \%)$ & \\
\hline Bilobectomy or pneumonectomy & $35(15 \%)$ & $22(15 \%)$ & $15(16 \%)$ & \\
\hline Approach & & & & .587 \\
\hline Open & $168(73 \%)$ & $106(71 \%)$ & $71(77 \%)$ & \\
\hline VATS or RVATS & $62(27 \%)$ & $43(29 \%)$ & $21(23 \%)$ & \\
\hline Length of stay, d & $5(4-6)$ & $4(3-6)$ & $4(3-6)$ & .006 \\
\hline Cardiopulmonary complications & $94(41 \%)$ & $51(34 \%)$ & $23(25 \%)$ & .025 \\
\hline
\end{tabular}

ERAS, Enhanced recovery after surgery; FEVI, predicted forced expiratory volume in 1 second; ASA, American Society of Anesthesiologists; VATS, video-assisted thoracic surgery; RVATS, robotic-assisted thoracoscopic surgery. ${ }^{*} \mathrm{~N}(\%)$ or median (25th-75th percentile) unless otherwise indicated. $\dagger \mathrm{N}=28 \mathrm{missing}$. $\ddagger \mathrm{N}=13 \mathrm{missing}$.

\section{DISCUSSION}

Implementation of an enhanced recovery pathway was associated with facilitated delivery of adjuvant chemotherapy, with fewer delays after surgery to starting adjuvant chemotherapy and a higher rate of completing 4 or more cycles. Advanced age, worse preoperative performance status, and receiving a bilobectomy or pneumonectomy were also associated with decreased adjuvant chemotherapy use, in addition to tumor related factors. Last, the surgical approach, whether minimally invasive or open, was not associated with adjuvant chemotherapy use.
Ideally, to study the impact of ERAS pathways, we would randomize patients to usual care or ERAS. However, randomized trials are not feasible in all situations. At our institution, components of the ERAS pathway were trialed and refined by 2 faculty surgeons before full implementation. To proceed with a randomized trial, we would have been required to forego the transitional era or to randomize patients after having already trialed components of the ERAS pathway, risking a bias to the null. A baseline with preexisting ERAS features has been cited as the likely cause for at least 1 study to have not found improvement with 


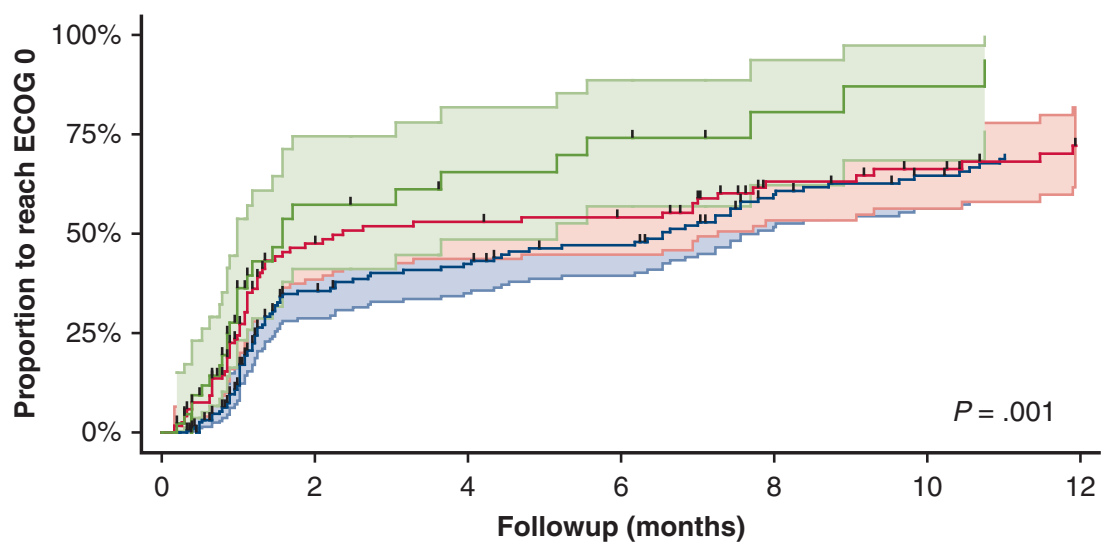

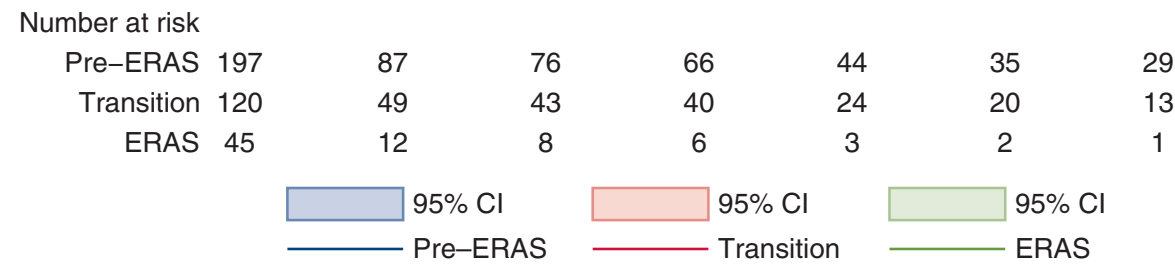

FIGURE 1. Time to ECOG performance status 0 after lung resection. ECOG, Eastern Cooperative Oncology Group; ERAS, enhanced recovery after surgery; $C I$, confidence interval.

ERAS implementation. ${ }^{3,23,24}$ Our presented analysis, using interrupted time series, represents a quasi-experimental design approach that takes full use of longitudinal data including preintervention trends and compares the change in trends before and after an intervention. ${ }^{14-17}$

As we have previously published, ${ }^{11}$ cardiopulmonary complications decreased during the enhanced recovery era. We also observed improved postoperative performance status after implementation of enhanced recovery, with patients showing an earlier time to reach ECOG performance status 0 during follow-up clinic visits. Similar results have been demonstrated after liver resection, for which an enhanced recovery pathway was associated with an earlier return to baseline functional status and improved completion of intended adjuvant oncologic therapy. ${ }^{25}$

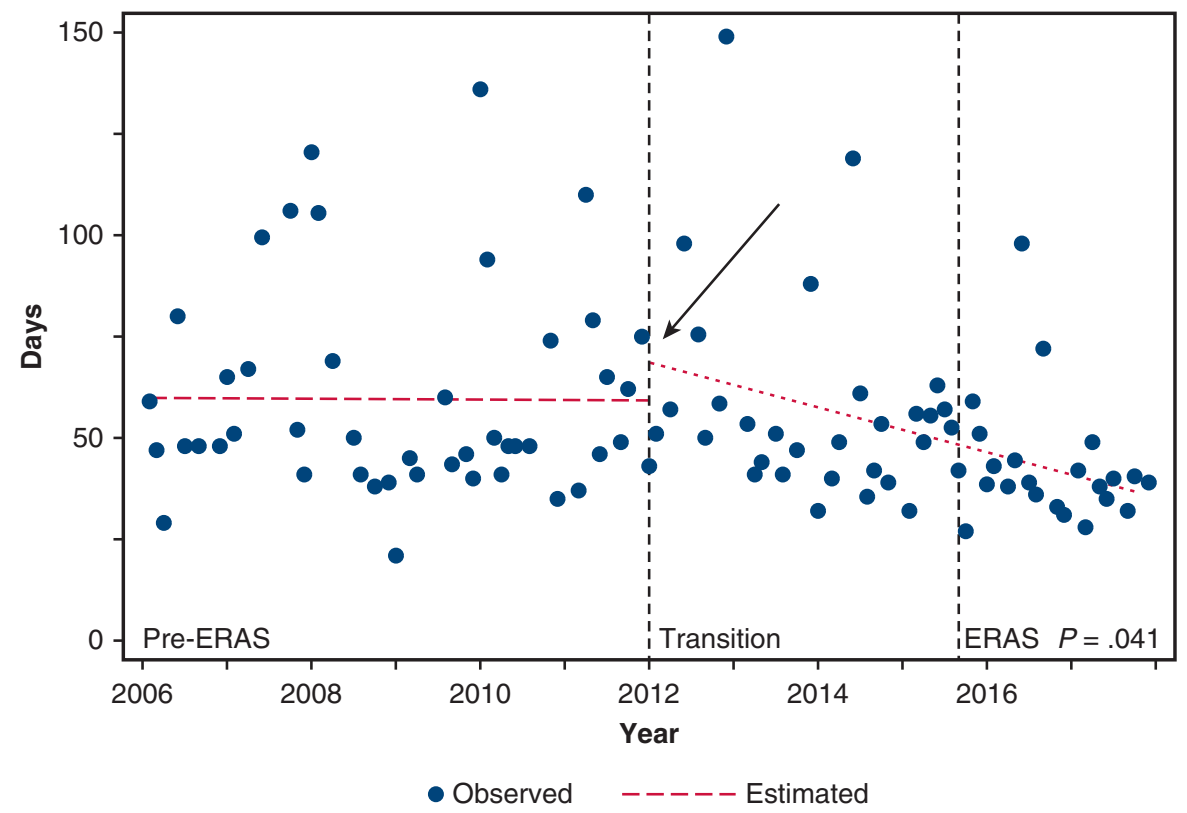

FIGURE 2. Interrupted time series for time to onset of adjuvant chemotherapy. Each dot indicates median time to onset of chemotherapy per month. Line indicates predicted trend during the time panel. Arrow indicates start of ERAS implementation. ERAS, Enhanced recovery after surgery. 
TABLE 3. Rate of completing adjuvant chemotherapy

\begin{tabular}{lcccc}
\hline & Pre-ERAS & Transition & ERAS & P value \\
\hline VATS/RVATS & 28 of $62(45 \%)$ & 20 of $43(47 \%)$ & 16 of $21(76 \%)$ & $.308^{*}$ \\
Open & 63 of $168(38 \%)$ & 55 of $106(52 \%)$ & 41 of $71(58 \%)$ & 57 of $92(62 \%)$ \\
Total & 91 of $230(40 \%)$ & 75 of $149(50 \%)$ & $<.001$ \\
\hline
\end{tabular}

ERAS, Enhanced recovery after surgery; VATS, video-assisted thoracic surgery; RVATS, robotic-assisted thoracoscopic surgery. *Mantel-Haenszel test for heterogeneity between VATS/RVATS and open.

Thoracotomy has not been consistently shown to interfere with adjuvant chemotherapy. Petersen and colleagues ${ }^{26}$ revealed that thoracotomy was associated with a delay to initiation of chemotherapy and a reduction in doses compared with VATS. Other retrospective series have shown similar results. ${ }^{27-29}$ However, Licht and colleagues ${ }^{30}$ also showed in a large Dutch series that adjuvant chemotherapy use was independently associated with age, comorbidity index, and pN2 status, but not with surgical approach. Our results support those of Licht and colleagues in showing no association between thoracotomy and failure to receive adjuvant chemotherapy.

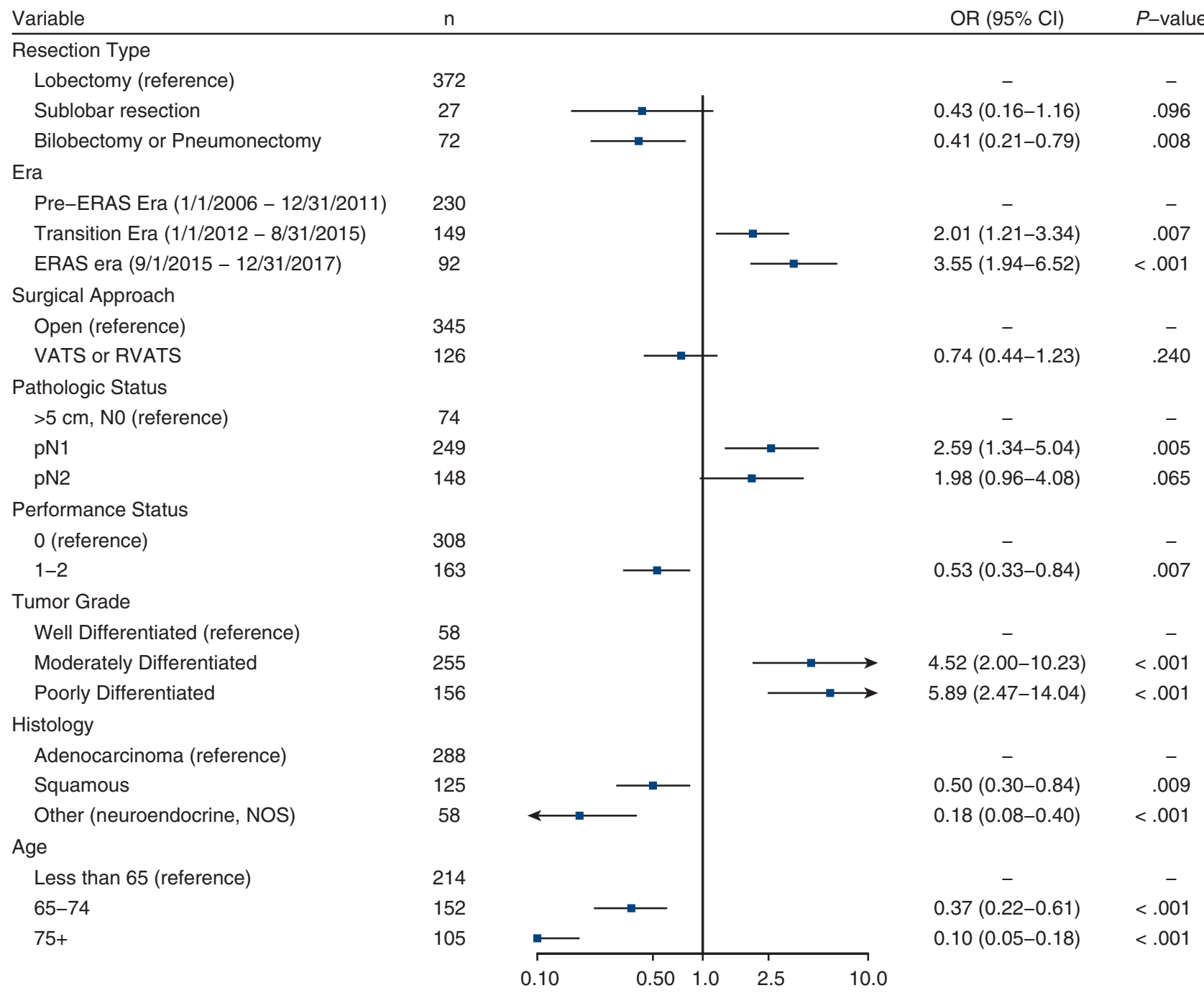

Less Likely to Complete Adjuvant Chemotherapy More Likely to Complete Adjuvant Chemotherapy

FIGURE 3. Multiple logistic regression associated with receiving adjuvant chemotherapy. ERAS, Enhanced recovery after surgery; VATS, video-assisted thoracoscopic surgery; RVATS, robotic-assisted thoracoscopic surgery; NOS, not otherwise specified. 
Enhanced recovery may provide a possible explanation for this finding. We have previously shown morbidity to be decreased among patients who receive thoracotomy after implementation of an enhanced recovery program, ${ }^{11}$ which has also been replicated by other centers. ${ }^{8}$ Of note, the same group that was unable to identify an association between thoracotomy and impaired adjuvant chemotherapy use also found no significant differences in postoperative morbidity between VATS and open lobectomy in a randomized trial. ${ }^{31}$ It may be useful to revisit the question of whether thoracoscopic surgery facilitates delivery of adjuvant chemotherapy in light of our results and those of Licht and colleagues. ${ }^{30}$

\section{Study Limitations}

Limitations of this retrospective analysis include that increased adjuvant chemotherapy completion from the pre-ERAS to ERAS era could be explained by trends related to changes in provider or patient preferences over time, or other confounding factors. We were unable to collect the reason for failing complete adjuvant chemotherapy for this study. Our study period was selected because adjuvant chemotherapy was highly encouraged by all practitioners at our institution during the study duration. Although the LACE trial was published in $2008,{ }^{32}$ many prior randomized trials showed an overall survival benefit for adjuvant chemotherapy. A recent analysis using the National Cancer Database revealed that adjuvant chemotherapy use nationwide has remained steady, approximately $40 \%$ from 2006 onward for stage II-IIIA disease, and has qualitatively decreased from $25 \%$ to $10 \%$ for stage IB disease. ${ }^{33}$ Unfortunately, the last year of data collection for their study was 2011. To the authors' knowledge, there were no major trials published during our study period to indicate the preferred chemotherapy regimen would change or to improve the quality of evidence toward adjuvant chemotherapy. Our statistical approach also showed that the trend in time to receiving chemotherapy was stable until an inflection point occurred in 2012. Nonetheless, other factors may impact adjuvant chemotherapy use, and a comparative analysis between centers that have implemented ERAS versus those who have not may help provide further clarity with regard to trends. Second, although our study encompasses 471 patients, we may be underpowered to detect a difference, especially among subgroup analysis. Third, postoperative performance status that was collected during the study duration was unblinded, introducing a possible observer bias. Last, our results represent those of a high-volume tertiary care center, which may limit generalizability.

\section{CONCLUSIONS}

Implementation of the ERAS pathway was associated with improved adjuvant chemotherapy delivery, with an earlier onset to start adjuvant chemotherapy and an increased rate of completing 4 or more cycles of adjuvant chemotherapy observed before and after our enhanced recovery program was implemented. As adjuvant chemotherapy has been demonstrated in substantial number of randomized trials to improve survival for NSCLC, our results suggest that ERAS, in addition to reducing postoperative complications and hastening postoperative recovery, may help return patients back to important oncologic therapy.

\section{Conflict of Interest Statement}

Authors have nothing to disclose with regard to commercial support.

\section{References}

1. Allen MS, Darling GE, Pechet TTV, Mitchell JD, Herndon Ii JE, Landreneau RJ, et al. Morbidity and mortality of major pulmonary resections in patients with early-stage lung cancer: initial results of the randomized, prospective ACOSOG Z0030 trial. Ann Thorac Surg. 2006;81:1013-20.

2. Spanjersberg WR, Reurings J, Keus F, van Laarhoven CJ. Fast track surgery versus conventional recovery strategies for colorectal surgery. Cochrane Database Syst Rev. 2011;Cd007635.

3. Brunelli A. Enhanced recovery pathway versus standard care in patients undergoing video-assisted thoracoscopic lobectomy. J Thorac Cardiovasc Surg. 2017;154:2084-90

4. Fiore JF Jr, Bejjani J, Conrad K, Niculiseanu P, Landry T, Lee L, et al. Systematic review of the influence of enhanced recovery pathways in elective lung resection. J Thorac Cardiovasc Surg. 2016;151:708-15.e706.

5. Scarci M, Solli P, Bedetti B. Enhanced recovery pathway for thoracic surgery in the UK. J Thorac Dis. 2016;8:S78-83.

6. Numan RC, Klomp HM, Li W, Buitelaar DR, Burgers JA, Van Sandick JW, et al A clinical audit in a multidisciplinary care path for thoracic surgery: an instrument for continuous quality improvement. Lung Cancer. 2012;78:270-5.

7. Salati M, Brunelli A, Xiumè F, Refai M, Pompili C, Sabbatini A. Does fasttracking increase the readmission rate after pulmonary resection? A casematched study $\dagger$. Eur J Cardiothorac Surg. 2012;41:1083-7.

8. Muehling BM, Halter GL, Schelzig H, Meierhenrich R, Steffen P, SunderPlassmann L, et al. Reduction of postoperative pulmonary complications after lung surgery using a fast track clinical pathway. Eur J Cardiothorac Surg. 2008;34:174-80

9. Das-Neves-Pereira JC, Bagan P, Coimbra-Israel AP, Grimaillof-Junior A, CesarLopez G, Milanez-de-Campos JR, et al. Fast-track rehabilitation for lung cance lobectomy: a five-year experience. Eur J Cardiothorac Surg. 2009;36:383-92.

10. Madani A, Fiore JF Jr, Wang Y, Bejjani J, Sivakumaran L, Mata J, et al. An enhanced recovery pathway reduces duration of stay and complications after open pulmonary lobectomy. Surgery. 2015;158:899-910.

11. Van Haren RM, Mehran RJ, Correa AM, Antonoff MB, Baker CM, Woodard TC, et al. Enhanced recovery decreases pulmonary and cardiac complications following thoracotomy for lung cancer. Ann Thorac Surg. 2018;106:272-9.

12. Rice DC, Cata JP, Mena GE, Rodriguez-Restrepo A, Correa AM, Mehran RJ Posterior intercostal nerve block with liposomal bupivacaine: an alternative to thoracic epidural analgesia. Ann Thorac Surg. 2015;99:1953-60.

13. Rogers LJ, Bleetman D, Messenger DE, Joshi NA, Wood L, Rasburn NJ, et al. The impact of enhanced recovery after surgery (ERAS) protocol compliance on morbidity from resection for primary lung cancer. J Thorac Cardiovasc Surg. 2018;155:1843-52.

14. Longtin Y, Paquet-Bolduc B, Gilca R, Garenc C, Fortin E, Longtin J, et al. Effec of detecting and isolating Clostridium difficile carriers at hospital admission on the incidence of $\mathrm{C}$ difficile infections: a quasi-experimental controlled study. JAMA Intern Med. 2016;176:796-804.

15. Kontopantelis E, Doran T, Springate DA, Buchan I, Reeves D. Regression based quasi-experimental approach when randomisation is not an option: interrupted time series analysis. BMJ. 2015;350.

16. Wagner AK, Soumerai SB, Zhang F, Ross-Degnan D. Segmented regression analysis of interrupted time series studies in medication use research. J Clin Pharm Ther. 2002;27:299-309. 
17. Grijalva CG, Nuorti JP, Arbogast PG, Martin SW, Edwards KM, Griffin MR. Decline in pneumonia admissions after routine childhood immunisation with pneumococcal conjugate vaccine in the USA: a time-series analysis. Lancet. 2007;369:1179-86.

18. Booth CM, Shepherd FA, Peng Y, Darling G, Li G, Kong W, et al. Time to adjuvant chemotherapy and survival in non-small cell lung cancer: a populationbased study. Cancer. 2013;119:1243-50.

19. Salazar MC, Rosen JE, Wang Z, Arnold BN, Thomas DC, Herbst RS, et al. Association of delayed adjuvant chemotherapy with survival after lung cancer surgery. JAMA Oncol. 2017;3:610-9.

20. Guidelines N. National Comprehensive Cancer Network Clinical Practice Guidelines in Oncology: Non-Small Cell Lung Cancer. Available at: https://www.ncen. org/professionals/physician_gls/pdf/nscl.pdf. Accessed April 3, 2018.

21. Mehran RJ, Martin LW, Baker CM, Mena GE, Rice DC. Pain management in an enhanced recovery pathway after thoracic surgical procedures. Ann Thorac Surg. 2016;102:e595-6.

22. Linden A. Conducting interrupted time-series analysis for single- and multiplegroup comparisons. Stata J. 2015;15:480-500.

23. Cerfolio RJ. Call it "fast tracking" or "enhanced recovery pathways" - No matter the name, it ain't nothing new to thoracic surgeons. J Thorac Cardiovasc Surg. 2017; 154:2091.

24. Kidane B. A rose by any other name: marginal gains of enhanced recovery after surgery in video-assisted thoracic surgery perioperative care. J Thorac Cardiovasc Surg. 2017;154:2082-3.

25. Day RW, Cleeland CS, Wang XS, Fielder S, Calhoun J, Conrad C, et al. Patientreported outcomes accurately measure the value of an enhanced recovery program in liver surgery. J Am Coll Surg. 2015;221:1023-30.e1022.

26. Petersen RP, Pham D, Burfeind WR, Hanish SI, Toloza EM, Harpole DH Jr, et al. Thoracoscopic lobectomy facilitates the delivery of chemotherapy after resection for lung cancer. Ann Thorac Surg. 2007;83: 1245-50.

27. Teh E, Abah U, Church D, Saka W, Talbot D, Belcher E, et al. What is the extent of the advantage of video-assisted thoracoscopic surgical resection over thoracotomy in terms of delivery of adjuvant chemotherapy following non-small-cell lung cancer resection? Interactive cardiovascular and thoracic surgery. 2014;19:656-60.

28. Jiang G, Yang F, Li X, Liu J, Li J, Zhao H, et al. Video-assisted thoracoscopic surgery is more favorable than thoracotomy for administration of adjuvant chemotherapy after lobectomy for non-small cell lung cancer. World J Surg Oncol. 2011;9.

29. Lee JG, Cho BC, Bae MK, Lee CY, Park IK, Kim DJ, et al. Thoracoscopic lobectomy is associated with superior compliance with adjuvant chemotherapy in lung cancer. Ann Thorac Surg. 2011;91:344-8.

30. Licht PB, Schytte T, Jakobsen E. Adjuvant chemotherapy compliance is not superior after thoracoscopic lobectomy. Ann Thorac Surg. 2014;98:411-6.

31. Bendixen M, Jorgensen OD, Kronborg C, Andersen C, Licht PB. Postoperative pain and quality of life after lobectomy via video-assisted thoracoscopic surgery or anterolateral thoracotomy for early stage lung cancer: a randomised controlled trial. Lancet Oncol. 2016;17:836-44.

32. Pignon JP, Tribodet H, Scagliotti GV, Douillard JY, Shepherd FA, Stephens RJ, et al. Lung adjuvant cisplatin evaluation: a pooled analysis by the LACE Collaborative Group. J Clin Oncol. 2008;20:3552-9.

33. Rajaram R, Paruch JL, Mohanty S, Holl JL, Bilimoria KY, Ko CY, et al. Patterns and predictors of chemotherapy use for resected non-small cell lung cancer. Ann Thorac Surg. 2016;101:533-40.

Key Words: enhanced recovery, surgery, lung cancer, adjuvant chemotherapy 


\section{APPENDIX E1. CHARACTERISTICS OF PROTOCOLIZED PATIENT CARE DURING EACH ERA \\ Pre-ERAS Era (January 1, 2006, to December 31, 2011)}

At the preoperative visit, patients were provided with videos that gave general postoperative information. All patients were required to fast for a minimum of 8 hours before surgery. No preventive analgesia medications were administered before surgery. A thoracic epidural anesthesia (TEA) catheter was placed in the T4-T8 region before induction of anesthesia, and catheter infusion was started 30 minutes before anesthesia emergence. A local anesthetic $(0.075 \%$ or $1 \%$ bupivacaine) and opioid (fentanyl at $15 \mathrm{mg} / \mu \mathrm{L}$ or hydromorphone at $10 \mathrm{mg} / \mu \mathrm{L}, 2 \mathrm{mg} / \mu \mathrm{L}$ ) were typically used for the epidural catheter. We did not use intraoperative opioid-sparing strategies or total intravenous anesthesia. Fixed fluid replacement, based on estimated blood loss and insensible loss, was used to guide fluid replacement. Two $28 \mathrm{~F}$ to $32 \mathrm{~F}$ chest tubes were typically inserted and removed after less than $300 \mathrm{~mL} /$ 24 hours. Postoperative pain was controlled with TEA or patient-controlled analgesia with fentanyl or hydromorphone. Patients were routinely ambulating on the day of surgery. Ambulation or out of bed to chair was encouraged at least 4 times daily. Patients were given clear liquids on postoperative day 1 , and the diet was advanced as tolerated.

\section{Transitional Era (January 1, 2012, to August 31, 2015)}

A multidisciplinary ERAS committee was formed to implement an ERAS pathway by using evidence-based bundled practices. The committee included representatives from thoracic surgery, anesthesia, nursing, and clinical pharmacology. During this transitional era, components of the ERAS pathway were trialed and refined. These included preemptive analgesia, liberalization of preoperative fasting, and alternatives to TEA, including intercostal nerve blocks, total intravenous analgesia during the intraoperative period, intravenous steroids, goal-directed fluid therapy, opioidsparing multimodal analgesia, and chest tube management. Two faculty surgeons (D.C.R. and R.J.M.) initially implemented these components, which were subsequently adopted by other faculty.
ERAS Era (September 1, 2015, to December 31, 2017)

The final version of the ERAS pathway was uniformly implemented by all surgeons for all major thoracic procedures on September 1, 2015. This is summarized in Table 1. Additional preoperative teaching instructing about ERAS principles, patient and caregiver expectations, and pain management were provided. Clear liquids were permitted up to 2 hours before scheduled surgery. Preventive analgesia with tramadol extended-release at $300 \mathrm{mg}$ orally and gabapentin at $300 \mathrm{mg}$ orally was administered on patient arrival in the preoperative holding area. Dexamethasone at $10 \mathrm{mg}$ intravenously (IV) was given on induction of anesthesia. Intraoperative opioids were spared, with acetaminophen at $1000 \mathrm{mg}$ IV given at induction and ketorolac at $30 \mathrm{mg}$ IV given 30 minutes before emergence from anesthesia. Propofol and dexmedetomidine were administered IV without routine use of volatile inhalational anesthetics.

Fluid replacement was guided according to a validated algorithm with noninvasive pulse wave monitoring of stroke volume. TEA was avoided, and regional anesthesia was administered with a preincisional posterior intercostal nerve blockade and local wound infiltration with $266 \mathrm{mg}$ of longacting liposomal bupivacaine. Typically, a single chest tube, $20 \mathrm{~F}$ to $24 \mathrm{~F}$, was inserted and then removed after less than $400 \mathrm{~mL} / 24$ hours of drainage. A standard algorithm for multimodal postoperative analgesia was used, which consisted of the following: $1000 \mathrm{mg}$ of IV acetaminophen for 48 hours followed by per os, $15 \mathrm{mg}$ of IV ketorolac for 48 hours, followed by oral nonsteroidal anti-inflammatory drugs and then $300 \mathrm{mg}$ of oral gabapentin. Breakthrough pain was controlled first with $50 \mathrm{mg}$ of oral tramadol followed by oral or intravenous opioids as needed (sustained pain score $\geq 4 / 10$ ). Early ambulation was encouraged as before. Patients were allowed clear liquids on postoperative day 0 , and diet was advanced as tolerated.

The ERAS pathway was modified in certain populations. Nonsteroidal anti-inflammatory drugs were not administered to those with a history of nephrectomy or a serum creatinine of greater than $1.5 \mathrm{mg} / \mathrm{dL}$. Gabapentin was reduced to $100 \mathrm{mg} 3$ times daily for those who experienced neurologic symptoms, including lightheadedness or tremors. Abbreviated fasting was avoided in patients who were considered to be at risk of aspiration, including those with a history of head or neck irradiation, esophageal stricture, achalasia, or vocal cord paresis. 